\title{
Wound healing: a new perspective on glucosylated tetrahydrocurcumin
}

This article was published in the following Dove Press journal:

Drug Design, Development and Therapy

13 July 2015

Number of times this article has been viewed

\author{
Adari Bhaskar Rao' \\ Ernala Prasad' \\ Seelam Siva Deepthi' \\ Vennapusa Haritha' \\ Sistla Ramakrishna' \\ Kuncha Madhusudan' \\ Mullapudi Venkata Surekha ${ }^{2}$ \\ Yerramilli Sri Rama \\ Venkata Rao ${ }^{3}$ \\ 'Medicinal Chemistry and \\ Pharmacology Division, Council \\ of Scientific and Industrial \\ Research - Indian Institute of \\ Chemical Technology, ${ }^{2}$ Pathology \\ Division, National Institute of \\ Nutrition, ${ }^{3}$ Ashian Herbex Ltd, \\ Hyderabad, AP, India
}

\begin{abstract}
Wound healing represents a dynamic set of coordinated physiological processes observed in response to tissue injury. Several natural products are known to accelerate the process of wound healing. Tetrahydrocurcumin (THC), an in vivo biotransformed product/ metabolite of curcumin, is known to exhibit a wide spectrum of biological activities similar to those of native curcuminoids. The poor bioavailability of these curcuminoids limits their clinical applications. The present study highlights the percutaneous absorption and wound healing activity of glucosyl-conjugated THC (glucosyl-THC) in male Wistar rats. A high plasma concentration of glucosyl-THC $(4.35 \mu \mathrm{g} / \mathrm{mL})$ was found in rats 3 hours after application. A significant enhanced wound healing activity and reduced epithelialization time were observed in rats that received glucosyl-THC. This may have been due to the improved bioavailability of the glucosyl compound. The nonstaining and lack of skin-sensitive side effects render the bioconjugated glucosyl-THC a promising therapeutic compound in the management of excision wounds and in cosmetic applications, in the near future.
\end{abstract}

Keywords: glucosylation, epithelialization, granulation tissue, cosmetic, therapeutic

\section{Introduction}

Natural products isolated from plants and microbes are major sources for exploration of novel pharmacophores of biological interest (drugs and agrochemicals), and more than $30 \%$ of the current worldwide sales of drugs are based on natural products. ${ }^{1,2}$ The phytochemicals in general continue to serve in the design of new synthetic compounds of therapeutic interest (pharmaceutical or cosmetic) against various diseases. ${ }^{3}$

The skin is the outer covering of the living body, consisting of multiple layers of the ectodermal tissues guarding the underlying muscles, bones, ligaments, and internal organs. It also provides protection against heat, light, infection, and injury. ${ }^{4,5}$ Wound healing refers to the complex and multifactorial process in response to a tissue injury, involving the cellular phase (granulation), narrowing of wound area (wound contraction), collagen deposition (collagen formation), epithelial covering (epithelialization), and scar remodeling (cicatrization). The smooth progression of all these events leads to a successful completion of wound healing, and restoration of the disrupted anatomical continuity and functional state of the skin. ${ }^{6-9}$

Phenolic compounds of natural origin have been shown to possess potent antioxidant activity and are also known to accelerate the process of wound healing. ${ }^{10-12}$ Among such phytochemicals, the curcuminoids present in the rhizomes of Curcuma longa have attracted a lot of attention due to their wide application in traditional medications, food coloration, cosmetics, and the fabric-dying industry. ${ }^{13,14}$ In addition, curcuminoids possess a broad range of therapeutic activities, such as anticancer, antiangiogenic, antimicrobial, antioxidant, anti-inflammatory, and wound healing properties. ${ }^{15-21}$
Correspondence: Adari Bhaskar Rao Medicinal Chemistry and Pharmacology Division, Council of Scientific and Industrial Research - Indian Institute of Chemical Technology, Tarnaka, Hyderabad 500 007, Telangana, India Tel +9l 27193374

Email adarirao2002@yahoo.co.in 
Tetrahydrocurcumin (THC) is one of the major in vivo colorless hydrogenated metabolites of curcumin and shows similar physiological and pharmacological activities to that of native curcuminoids. $^{22-24}$ Despite the unique biological activities of these curcuminoids, the major barrier for wider clinical applications of these molecules is their poor bioavailability in the systemic circulation in humans, which reduces their therapeutic activity. Curcuminoids have very poor aqueous solubility and permeability, and so these molecules are classified as Biopharmaceutics Classification System (BCS) class IV molecules. ${ }^{25}$ Several efforts have been made to increase the bioavailability of curcumin/THC with different formulations, using adjuvants, nanoparticles, and liposomes etc, which have resulted in a limited success. ${ }^{26-29}$ Another method to improve the bioavailability and pharmacological activities is the glucosylation of the hydrophobic molecule. ${ }^{30,31}$ The objective of the present study was to evaluate the in vivo percutaneous absorption and wound healing activity of synthesized glucosyl-conjugated THC (glucosyl-THC) in Wistar rats.

\section{Materials and methods}

\section{Chemicals}

Curcumin (98\%) and THC (90\%) (analyzed using highperformance liquid chromatography [HPLC]) were gift samples obtained from Ashian Herbex Ltd (Hyderabad, India). Standard curcumin (catalog number 1151855) and THC (catalog number SM1300370) were procured from SigmaAldrich Corp (St Louis, MO, USA). Glucosyl-THC was synthesized as reported in our earlier studies. ${ }^{32}$ All the chemicals and solvents used in the study were of analytical grade and purchased from Merck Millipore (Billerica, MA, USA).

\section{Experimental animals}

Male albino Wistar rats, weighing 150-200 g (8-10 weeks), were selected for the study and were daily provided with commercial feed (Hindustan Lever, Bangalore, India) and water ad libitum. The experimental rats used and the protocols followed in this study were reviewed and approved by the Institutional Animal Ethics Committee prior to the initiation of the experiment. The care of the laboratory animals was guided by the Committee for the Purpose of Control and Supervision of Experiments on Animals (CPCSEA) regulations.

\section{Topical/percutaneous absorption studies}

Percutaneous absorption studies of the curcuminoids (curcumin, THC, and glucosyl-THC) in experimental rats were carried out as reported by Van de Sandt et al. ${ }^{33}$ Male Wistar rats weighing 180-200 g were randomly divided into five groups with five animals in each group. On the day of the treatment, the fur on the dorsal upper side of the experimental rats was shaved $\left(2 \mathrm{~cm}^{2}\right)$, with a disposable razor, and the area was cleaned and sterilized with alcohol (40\%). Each of the experimental rats received an application of $1 \mathrm{~g}$ of petroleum jelly or cream containing different concentrations of curcuminoids: group A received petroleum jelly alone; group B received $1 \%$ curcumin; group $\mathrm{C}$ received $1 \% \mathrm{THC}$; and groups $\mathrm{D}$ and $\mathrm{E}$ received cream containing $1 \%$ and $2 \%$ glucosyl-THC respectively, (approximately $15 \mathrm{~cm}^{2} / \mathrm{kg}$ body weight). The cream was topically applied, uniformly, on the dorsal skin area of the rats, and the surface was covered with a protective elastic adhesive bandage (as per the Organisation for Economic Co-operation and Development [OECD] Guideline for the Testing of Chemicals - Skin Absorption: In Vivo Method).

Rat blood samples $(0.2 \mathrm{~mL})$ were drawn from the retroorbital vein at different time intervals ( $0-8$ hours) into glass tubes containing ethylenediaminetetraacetic acid (EDTA). After centrifugation $(1,000 \times g$ for 10 minutes at $5^{\circ} \mathrm{C}$ ), the separated plasma samples were stored at $-20^{\circ} \mathrm{C}$ for analysis. Salbutamol $(0.2 \mu \mathrm{g} / \mathrm{mL})$ was added to all the samples as an internal standard. The test compounds curcumin, THC, and glucosyl-THC - present in the plasma samples were extracted twice using organic solvents (ethyl acetate and ethanol [9.5:0.5]). The organic layer was transferred into clean tubes. Standard concentrations $(50 \mu \mathrm{g} / \mathrm{mL})$ of curcuminoids (curcumin, THC, and glucosyl-THC) were added, as external standards, to respective test samples for spiking of the samples. Later, the organic solvent was concentrated under a stream of dry nitrogen. The concentrated samples thus obtained were dissolved in the HPLC mobile phase and filtered through $0.22 \mu \mathrm{M}$ polyvinylidene difluoride membrane filters (Merck Millipore) to obtain clear filtrate for HPLC and liquid chromatography mass spectrometry (LC-MS) analysis. ${ }^{34}$ The quantification of the curcuminoids studied was accomplished by measuring the peak area ratio (test compound to internal standard). This process of compound extraction gives a quantitative validation; the results showed that approximately $95 \%$ of the curcuminoids present in the plasma samples were recovered.

\section{Data analysis HPLC}

The concentration of curcuminoids (curcumin, THC, and glucosyl-THC) present in the plasma samples was analyzed by HPLC (Gilson Company, Inc., Middleton, WI, USA) using 
a reverse-phase $\mathrm{C}_{18}$ analytical column $(3.9 \mathrm{~mm} \times 150 \mathrm{~mm}$, $5 \mu \mathrm{m}$ particle size). ${ }^{35}$ The system was run isocratically, using acetonitrile:water as a mobile phase in the ratio of $80: 20$ adjusted to $\mathrm{pH} 3.5$, at a flow rate of $1 \mathrm{~mL} / \mathrm{min}$. The compounds were detected at $280 \mathrm{~nm}$ by an ultraviolet-visible (UV/VIS) detector (UV/spectrophotometer from Perkin Elmer, Waltham, MA, USA). The mobile phase (solvent) was passed through a $0.5 \mu \mathrm{m}$ nylon membrane filter and was degassed before use. Salbutamol $(0.2 \mu \mathrm{g} / \mathrm{mL})$ was added to each of the test samples as an internal standard. The method shows sensitivity toward standard curcuminoids with quantization up to a lower limit of $1 \mathrm{ng} / \mathrm{mL}$.

\section{In vivo wound healing studies}

Excision wound

A cutaneous excision-punch wound model was studied to evaluate the wound healing activity of the three curcuminoids. An excision wound was inflicted on the rats' dorsal side as described in the literature. ${ }^{36}$ Before inflicting the wounds, the experimental rats were anesthetized by intraperitoneal administration of ketamine $(70 \mathrm{mg} / \mathrm{kg}$ body weight), and the fur on the dorsal side of the animals was shaved using a disposable razor and disinfected with $40 \%$ of ethanol. A uniform circular excision wound, of $500 \mathrm{~mm}^{2}$ and $0.2 \mathrm{~cm}$ depth, was created with a surgical blade on the dorsal surface at the thoracolumbar region of each of the experimental rats, under aseptic conditions. To each experimental rat, $1 \mathrm{~g}$ of petroleum jelly containing different concentrations of the test compounds (curcumin, THC, and glucosyl-THC) was topically applied, uniformly, whereas the control rats received petroleum jelly once daily for 21 days. Wounds were left unsutured and uncovered, and the animals were housed individually and allowed to recover. In order to characterize the various wound healing steps, several critical physical attributes of the wound healing process - inflammation, reepithelialization, wound dermal closure/contraction, epidermal differentiation, tissue remodeling, and eschar formation - were observed. The progress of wound healing was measured by tracing the wound on millimeter-scale graph paper after every 2 days of wound formation, and video images were also captured..$^{37}$ The epithelialization period was monitored by noting the number of days required for the eschar to fall off from the surface of the wound without leaving a raw area (scar) behind. A day after the final day of the treatment, the experimental rats were euthanized, and the regenerating granulation tissues from the excision wounds were dissected from the same area and processed for biochemical and histopathological studies.

\section{Dosage used in wound healing studies}

The effect of curcumin, THC, and glucosyl-THC on wound healing activity was studied in male Wistar rats. A total of 40 rats weighing 180-200 g were randomly selected and divided into eight groups consisting of five rats in each group. Rats in each of the different experimental groups topically received an application of $1 \mathrm{~g}$ of cream containing the following test compounds:

- Group 1: petroleum jelly as control

- Group 2: 2\% curcumin

- Groups 3, 4, and 5: 2\%, 3\%, and 5\% THC, respectively

- Groups 6, 7, and 8: 2\%, 3\%, and 5\% glucosyl-THC, respectively.

\section{Measurement of wound index}

Wound indices were measured after every 2 days of wound formation following a random scoring system. ${ }^{38}$ Wound healing property was evaluated as percentage of wound contraction, measuring the length and breadth of the wound with digital calipers following the Walker and Mason formula. ${ }^{39}$ Significance in wound healing of the test groups was derived by comparing the healed wound area, on the respective days, with the healed wound area of the control group. The rate of wound contraction was calculated using the given formula:

$$
\begin{gathered}
\% \text { wound } \\
\text { contraction }
\end{gathered}=\frac{\begin{array}{l}
\text { Initial area of wound }- \\
\text { nth day area of wound }
\end{array}}{\text { Initial area of wound }} \times 100
$$

\section{Biochemical parameters}

The granulation tissues were collected from the rat regenerating excision wounds and homogenized using $0.1 \mathrm{M}$ sodium phosphate buffer of $\mathrm{pH7}$.4. Total protein and collagen content present in the granulation tissue homogenate were analyzed.

\section{Total protein analysis}

The total protein concentration in the granulation tissue homogenate was estimated by Lowry's method. ${ }^{40}$

\section{Collagen analysis}

The total collagen content in the enzymatically digested granulation tissue collected from the experimental rats was determined using a Sircol ${ }^{\mathrm{TM}}$ assay kit (Biocolor, Carrickfergus, UK). ${ }^{41}$ The assay was based on the specific binding of the dye to collagen. This methodology gives only the collagen content, free from interference of noncollagenous protein (albumin and elastin). 


\section{Histopathology studies}

Histological changes, such as epithelialization, granulation tissue formation, and cell migration, were observed during the process of wound healing in individual experimental Wistar rats that received curcuminoids (curcumin, THC, and glucosyl-THC). On the 21 st day postwounding, the animals were sacrificed, and the granulation tissue formed on and around the excision wounds of the untreated and treated rats was carefully dissected with a sharp, sterile surgical knife $(0.4 \mathrm{~cm} \times 1.0 \mathrm{~cm})$ and carefully collected without any folding, and weighed. It was then fixed in $10 \%$ buffered formalin solution of $\mathrm{pH} 7.4$ and stored. After the usual processing of the tissue in dehydrated graded alcohol, these tissues were cleared in xylene and were embedded in paraffin wax (melting point $55^{\circ} \mathrm{C}$ ). Skin samples from three different wound healing sites were taken for histopathological studies. The skin tissue biopsies were cut into 5-6 $\mu \mathrm{m}$ thick sections and stained with hematoxylin and eosin. ${ }^{42}$ The sections were then observed under a light microscope (Eclipse 4000, Nikon Corp, Tokyo, Japan) for qualitative assessment of the degree of epithelialization, collagen formation, fibroblast proliferation, angiogenesis, and scar formation in the wound tissues of treated and untreated rats.

\section{Statistical analysis}

All the results depicted in the study were the mean \pm standard error (SE) of five rats in each group. Statistical analysis were evaluated by one-way analysis of variance (ANOVA), followed by Tuckey's post hoc test. The values of $* P<0.01$ and $* * P<0.001$ were considered to be statistically significant.

\section{Results and discussion}

Wound healing is a normal biological process in the human body that is achieved through a highly complex and intricate processes consisting of different phases, and these multifactorial processes are concurrent but are independent to each other in establishing the integrity of the damaged tissue. Several natural products have been shown to accelerate the process of wound healing. Curcuminoids are naturally occurring polyphenolic compounds well-known for their putative anti-inflammatory, antioxidant, and wound healing activities. This study demonstrates the topical absorption and wound healing property of glucosyl-THC in experimental rats.

\section{Topical/percutaneous absorption study}

The major barrier for clinical application of these curcuminoids is their poor solubility, which limits the bioavailability and therapeutic activity of these compounds in humans.
Bioenhancers, such as euganol, trienol, piperidine, failed to improve the bioavailability of curcuminoids. ${ }^{27}$ In comparison with two other curcuminoids studied, the $1 \%$ glucosyl-THC showed a higher plasma concentration, of $4.35 \mu \mathrm{g} / \mathrm{mL}$ at 3 hours peak time, on the topical application of the compounds to the rats (Figure 1). This may have been due to enhanced hydrophilicity of the glucosyl-conjugate molecule (LC-MS studies m/z [M + Na] ${ }^{+}$719.13).

\section{Wound healing studies}

The wound healing process is characterized by dynamic, interactive events involving soluble mediators, blood cells, extracellular matrix, and parenchymal cells that result in the permanent restoration of anatomic and functional integrity. ${ }^{43}$ In the present study, the excision wounds were made on dorsal side of rats, and the wounds were treated with curcumin, THC, and glucosyl-THC. From the results, it was observed that the animal behavior was normal during the entire experimental period, and the rats did not show any signs of compoundrelated toxicity or other related skin-sensitive side effects. The wound regions in both the treated and control rats were devoid of bleeding during the study period. Inflammation, redness, and erythema were observed in the initial days of wound in all the experimental animals. Acute inflammation was seen in the control group, while chronic inflammation was observed in curcuminoids-treated groups, which gradually decreased by the third day of treatment with glucosyl-THC, whereas in rats that received curcumin and THC, inflammation and erythema were reduced with time, ie, from day 5 to day 8 . Contraction of the excision wound was observed between 6 days and 9 days

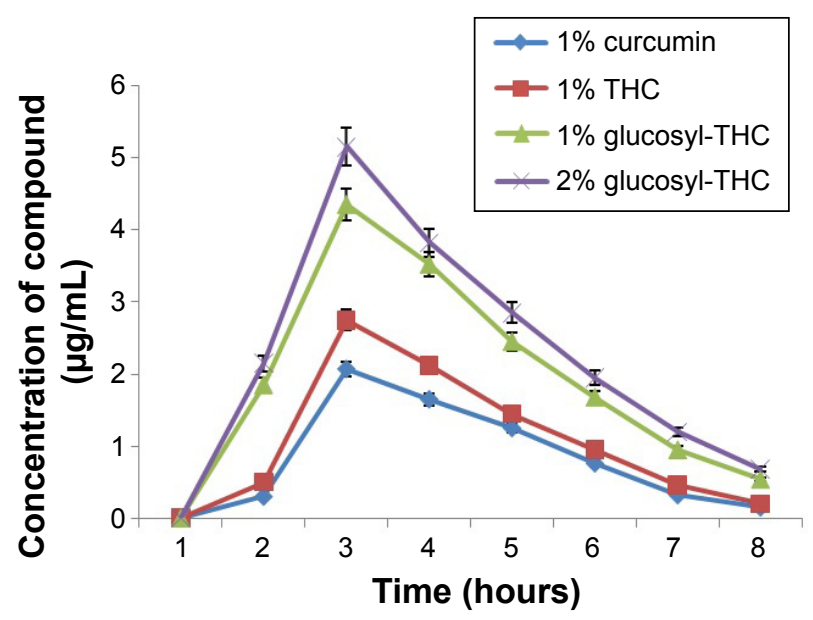

Figure I Percutaneous absorption of curcuminoids in rats (HPLC).

Notes: The values expressed by vertical bars represent mean \pm SE\% of five animals of each group.

Abbreviations: glucosyl-THC, glucosyl-conjugated tetrahydrocurcumin; HPLC, high-performance liquid chromatography; THC, tetrahydrocurcumin. 
in the rats that received curcumin, THC, and glucosyl-THC (Figure 2). An arbitrary scoring of wound healing is shown in Table 1. The three curcuminoids showed wound healing activity, without any abnormal behavior patterns, such as fatigue, stress, aggressiveness, weight loss, or change in movement, or signs of infection among the rats during the course of the study. Complete wound epithelialization with increased collagen formation was observed on the 18th day of wound healing in the experimental rats that received glucosyl-THC. The regenerated wound tissues treated with glucosyl-THC exhibited marked dryness, with no clinical signs of inflammation and fluids oozing from the wound margins.

When compared with curcumin and THC, the glucosylTHC-treated rats exhibited significantly faster closure of wounds. A significant wound healing activity, $88 \%$ $(P<0.001)$, was observed in $2 \%$ glucosyl-THC-treated rats, whereas in the rats that received curcumin and $\mathrm{THC}$, the percentage of wound healing was $76 \%(P<0.01)$ and $81 \%$ $(P<0.01)$, respectively (Figure 3$)$.

It is well-known that rapid protein synthesis occurs in the wound area soon after an injury. ${ }^{44}$ Collagen is the predominant extracellular protein in the granulation tissue of a healing wound, and the increase in synthesis of collagen provides strength and integrity to the tissue matrix. In the present study, the increased concentration of total protein was $9.2 \mathrm{mg} / 100 \mathrm{mg}$ and collagen content was $8.3 \mathrm{mg} / 100 \mathrm{mg}$ in the granulation tissue of the excision wounds in the samples treated with $2 \%$ glucosyl-THC when compared with other curcuminoids studied (Table 2).

The histopathological studies of the excision wound tissues of the rats that received glucosyl-THC showed morphological changes, such as granulation tissue formation, higher collagen content, and increased migration of macrophages and fibroblasts cells, which aid in granulation tissue formation and wound tissue repair (Figure 4). ${ }^{45,46}$ The histopathological scoring is given in Table S1. Visual observation confirmed that growth of the granulation tissue initiated from the base of the wound and proceeded to fill the entire wounds following treatment with both the THC and glucosyl-THC. The granulation tissue formed was pink-red in color, moist, and shiny. The percentage of wound contraction and reepithelialization after topically administered glucosyl-THC was promising when compared with the other two curcuminoids studied. This can be attributed to the
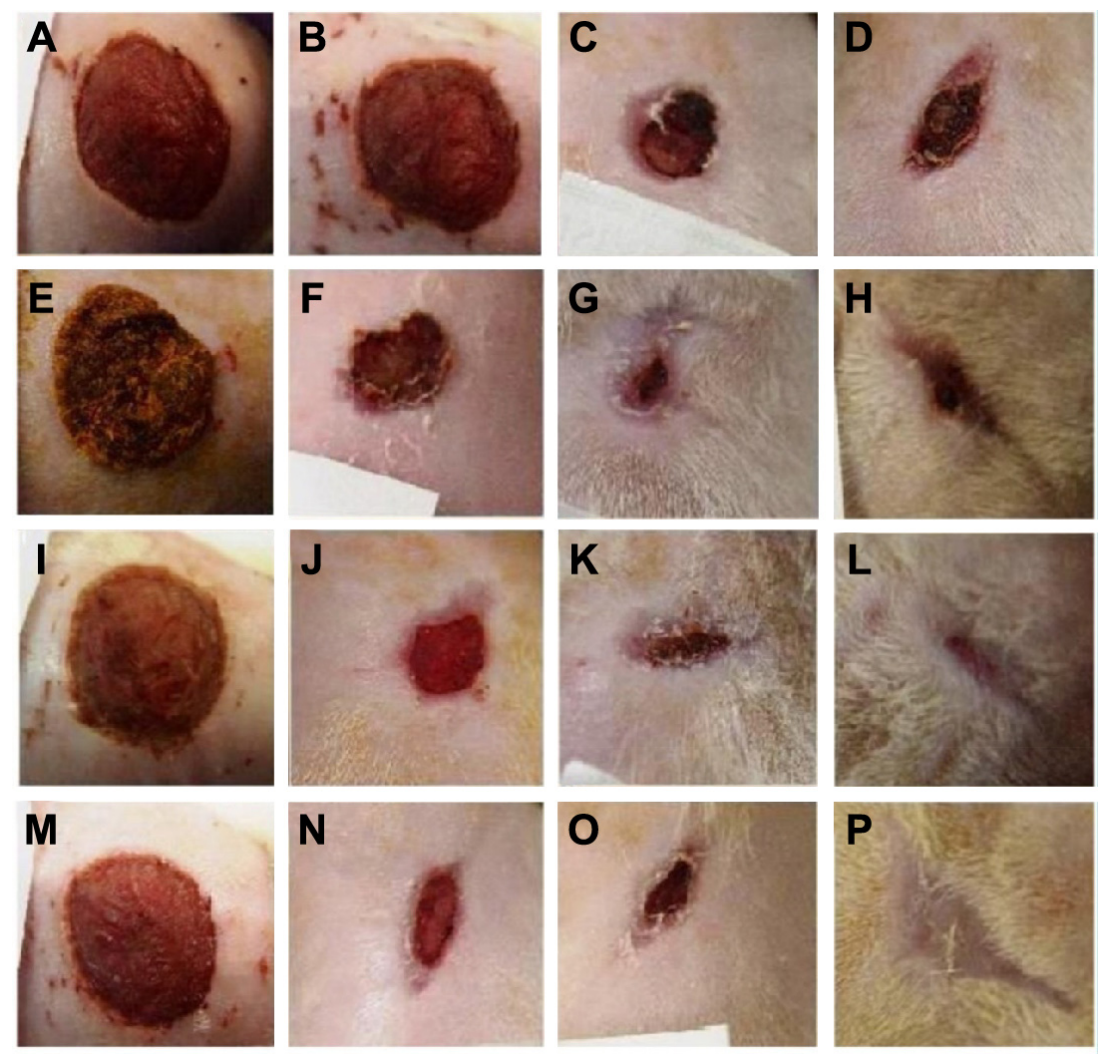

Figure 2 Photographical representation of wound healing in rats subjected to skin excision wounds, on different days (A,E,I,M after 2 days; B,F,J,N after 10 days; C,G,K,O after 15 days and $\mathbf{D}, \mathbf{H}, \mathbf{L}, \mathbf{P}$ after $2 \mathrm{I}$ days).

Notes: The rats received I g of topically applied cream containing: (A-D) petroleum jelly as control; (E-H) $2 \%$ curcumin; (I-L) $2 \%$ THC; and (M-P) $2 \%$ glucosyl-THC. Abbreviations: glucosyl-THC, glucosyl-conjugated tetrahydrocurcumin; THC, tetrahydrocurcumin. 
Table I A summary of wound healing index scores of rat models

\begin{tabular}{lllll}
\hline Treatment & 2 days after treatment & $\mathbf{7}$ days after treatment & I4 days after treatment & 2I days after treatment \\
\hline Control & Healing has not yet started & Healing has not yet started & Delayed but normal healing & Delayed but normal healing \\
Curcumin Std 2\% & Healing has not yet started & Delayed but normal healing & Incomplete but normal healing & Incomplete but normal healing \\
THC 2\% & Healing has not yet started & Delayed but normal healing & Incomplete but normal healing & Progressive but incomplete healing \\
Glucosyl-THC 2\% & Healing has not yet started & Incomplete but normal healing & Progressive and normal healing & Complete healing of wounds \\
\hline
\end{tabular}

Notes: Arbitrary scoring is given as follows: $0=$ complete healing of wounds, $\mathrm{I}=$ incomplete but normal healing, $2=$ delayed but normal healing, $3=$ healing has not yet been started, but the environment is healthy, $4=$ formation of pus, evidence of necrosis.

Abbreviations: glucosyl-THC, glucosyl-conjugated tetrahydrocurcumin; Std, standard; THC, tetrahydrocurcumin.

increased stimulation of collagen synthesis or increased proliferation of fibroblasts, or both, in the case of glucosyl-THCtreated wounds. The granulation tissue sections of the curcuminoid-treated animals showed significant adnexal and subepithelial collagen formation, whereas the animals in the control group showed less significant regenerative epithelium formation. It was observed that neovascularization was maximally seen in the early granulation tissue formation and was composed of proliferating blood vessels. ${ }^{47}$ Mature blood vessels accompanying collagen, fibrosis, and scar formation were observed in the wound healing activity of glucosyl-THC-treated rats. The same was evident in the

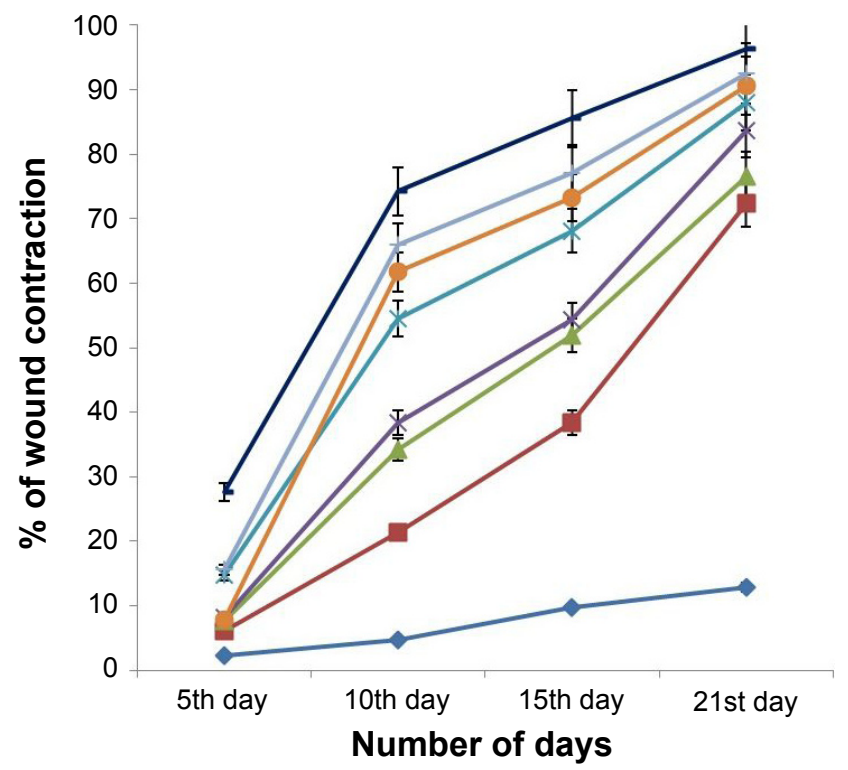

\begin{tabular}{ll|}
$\leftarrow$ Petroleum jelly & $-2 \%$ curcumin Std \\
$-2 \%$ THC & $\div 3 \%$ THC \\
$-5 \%$ THC & $-2 \%$ glucosyl-THC \\
$-3 \%$ glucosyl-THC & $-5 \%$ glucosyl-THC
\end{tabular}

Figure 3 Percentage of wound contraction in experimental rats.

Notes: The results depicted were the mean \pm SE\% of five animals in each group. The treated groups were compared with the control group, and statistical analysis was performed. The values of $P<0.01$ and $P<0.001$ were considered to be statistically significant.

Abbreviations: glucosyl-THC, glucosyl-conjugated tetrahydrocurcumin; SE, standard error; Std, standard; THC, tetrahydrocurcumin. histological wound scoring studies of epithelialization, granulation tissue formation, collagen organization, blood vessel formation, and polymorphonuclear leukocyte/macrophage migration. Fibrous scars were observed at the end of complete wound healing, and collagen was the predominant constituent (as shown in Table S1). ${ }^{47}$ Thus the study clearly demonstrated that the glucosyl-THC accelerated the process of wound healing compared with other curcuminoids, due its increased percutaneous absorption.

Since antiquity, curcumin has been used in the various traditional systems of medicine for various skin ailments, and preclinical studies have validated many ethnomedical uses. ${ }^{15-21} \mathrm{~A}$ few of the conducted human clinical trials showed protection from skin diseases such as psoriasis, prevention of aging, and promotion of good skin. ${ }^{48,49}$ However, detailed studies are required to validate the accepted belief.

The use of curcumin for chemoprevention and treatment of various skin diseases, such as scleroderma, psoriasis, and skin cancer, has been well-known. ${ }^{50}$ However, the important obstacle for transdermal drug delivery is the skin barrier. The present study confirms that all the curcuminoids tested show percutaneous absorption and wound healing activity. THC is a colorless metabolite of curcumin that exhibits pharmacological and biological activities similar to those of

Table 2 Biochemical parameters on the final day of granulation tissue formation in rats treated with curcuminoids

\begin{tabular}{lll}
\hline Group & $\begin{array}{l}\text { Protein } \\
\text { (mg/1 00 } \mathbf{~ m g})\end{array}$ & $\begin{array}{l}\text { Collagen } \\
\text { (mg/ 100 mg) }\end{array}$ \\
\hline Control (petroleum jelly) & $5.8 \pm 0.8$ & $2.12 \pm 0.54$ \\
Curcumin Std 2\% & $7.80 \pm 0.50^{*}$ & $6.7 \pm 0.81^{*}$ \\
THC 2\% & $8.8 \pm 0.9 *$ & $7.3 \pm 0.36^{*}$ \\
THC 3\% & $9.3 \pm 0.41^{*}$ & $8.5 \pm 0.42^{*}$ \\
THC 5\% & $9.9 \pm 0.38^{*}$ & $9.1 \pm 0.25^{* *}$ \\
Glucosyl-THC 2\% & $9.2 \pm 0.73^{*}$ & $8.3 \pm 0.41^{*}$ \\
Glucosyl-THC 3\% & $10.5 \pm 0.5 I^{*}$ & $9.4 \pm 0.78^{* *}$ \\
Glucosyl-THC 5\% & $10.9 \pm 0.83^{*}$ & $9.9 \pm 0.31^{* *}$ \\
\hline
\end{tabular}

Notes: All values expressed were mean \pm SE of five animals. Statistically significant from control $* P<0.01$ and $* * P<0.001$.

Abbreviations: glucosyl-THC, glucosyl-conjugated tetrahydrocurcumin; SE, standard error; Std, standard; THC, tetrahydrocurcumin. 

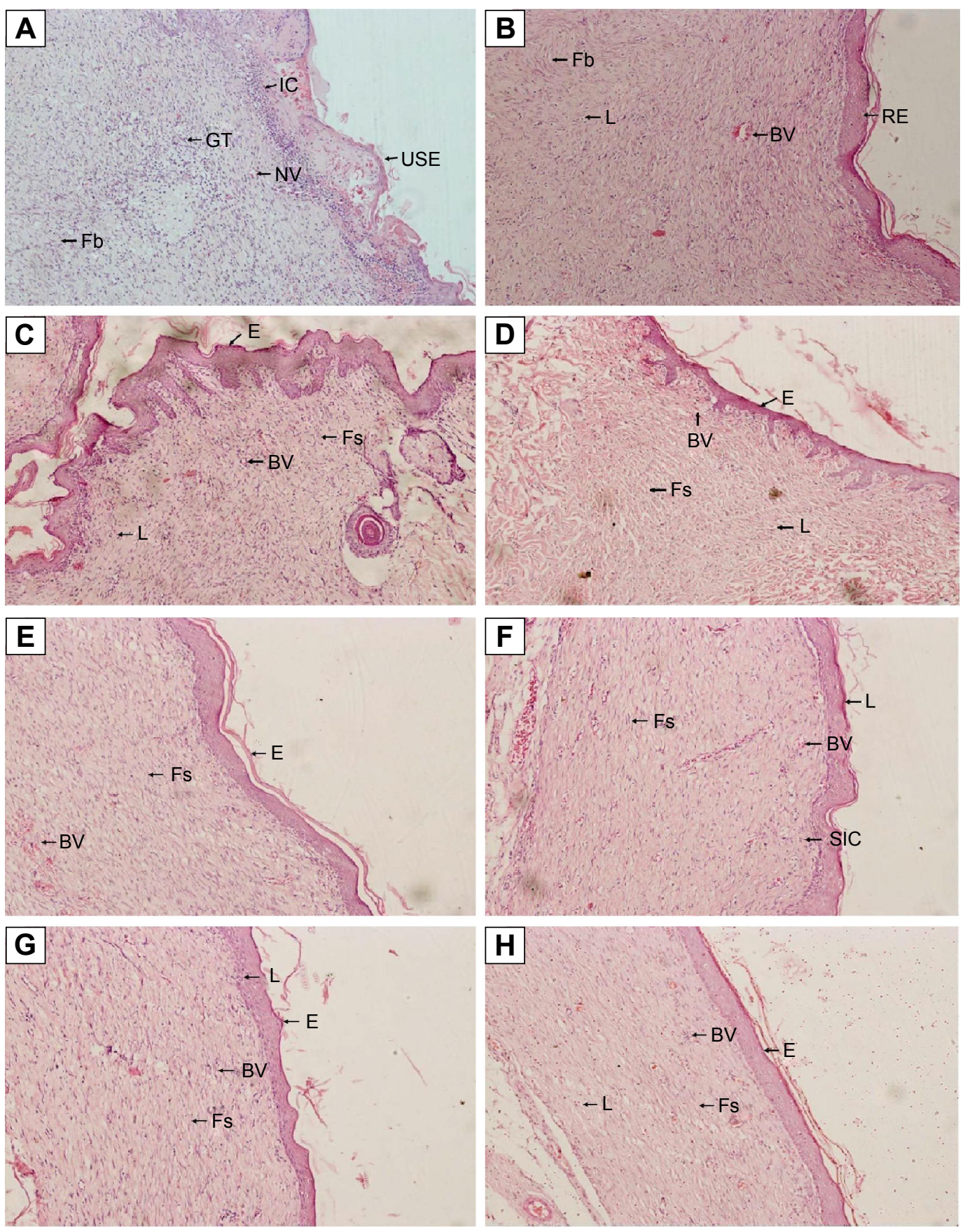

Figure 4 Microscopic observations representing the histological appearance of the granulation tissue in the excision wounds.

Notes: After the 21 st day of application of curcuminoids, rat skin tissue sections from excision wounds were stained with hematoxylin and eosin. All the slides were observed at 20× magnification: (A) control (placebo), (B) Standard (2\% curcumin Std), (C) $2 \%$ THC, (D) $3 \%$ THC, (E) $5 \%$ THC, (F) $2 \%$ glucosyl-THC, (G) $3 \%$ glucosyl-THC, and (H) $5 \%$ glucosyl-THC activity.

Abbreviations: BV, blood vessels; C, collagenation; E, epithelialization; Fb, fibroblasts; Fs, fibrosis; glucosyl-THC, glucosyl-conjugated tetrahydrocurcumin; GT, granulation tissue; IC, inflammatory cells; L, lymphocytes; NV, neovascularization; RE, reepithelialization; SIC, scattered inflammatory cells; Std, standard; THC, tetrahydrocurcumin; USE, ulcerated surface epithelium.

other curcuminoids. ${ }^{51}$ Compared with curcumin and THC, glucosyl-THC show significantly enhanced percutaneous absorption, due to improved hydrophilicity of the molecule. The glucosyl-THC also show improved wound healing activity in rats, and the histopathological studies showed reepithelialization of the epidermis, and increased migration of fibroblasts and macrophages in the wound bed. The most common drawback of the therapeutic use of the curcuminoids is their pronounced yellow color, resulting in staining of the skin and dress materials. The most significant advantage of 
the synthesized glucosyl-THC molecule is increased topical absorption, resulting in its enhanced wound healing activity with nonstaining property.

In summary the present study demonstrates increase in absorption of glucosyl-THC with topical application when compared with other curcuminoids. The results also confirm the improved pharmacological activity of glucosyl-THC, which was evident from the shorter wound healing time, increased rate of reepithelialization, and higher collagen formation in the granulation tissue in excision wound healing, and the absence of skin-sensitive side effects. The study clearly substantiates the medical value of glucosyl-THC in the acceleration of the process of wound healing when compared with other curcuminoids.

\section{Conclusion}

The wound healing process is characterized by three stages, viz inflammation, tissue formation, and tissue remodeling, which are coordinated cellular and biochemical responses. Plant secondary metabolites have been demonstrated as potential agents that can modify the various steps of wound repair. The present study confirms the increase percutaneous absorption and potent in vivo excision wound healing activity of glucosyl-THC. The compound glucosyl-THC increased the epithelialization and granulation tissue formation, with nonstaining, nontoxic, nonirritant, and non-skin-sensitive side effects. Thus the synthesized glucosyl-THC may act as a promising therapeutic agent in the future, in the management of excision wounds and in cosmetic applications.

\section{Acknowledgments}

Our sincere thanks to the Department of Science and Technology (DST), New Delhi, India, for the financial support of this project (grant number SR/SO/BB-54/2008). EP thanks DST for the award of a fellowship. The authors also thank the Managing Director, Ashian Herbix Ltd, Hyderabad, India, for the financial support.

The study is dedicated to Dr YVSS Murthy Vice Chairman of M/S Alkali Metals Ltd, Hyderabad, India.

\section{Disclosure}

The authors report no conflicts of interest in this work.

\section{References}

1. Grabley S, Thiericke R. The impact of natural products on drug discovery. In: Grabley S, Thiericke R, editors. Drug Discovery from Nature. Berlin: Springer-Verlag; 1999:3-37.

2. Helmstädter A, Staiger C. Traditional use of medicinal agents: a valid source of evidence. Drug Discov Today. 2014;19(1):4-7.
3. Biswas TK, Mukherjee B. Plant medicines of Indian origin for wound healing activity: a review. Int $J$ Low Extrem Wounds. 2003;2(1): $25-39$.

4. Kumar B, Vijayakumar M, Govindarajan R, Pushpangadan P. Ethnopharmacological approaches to wound healing - exploring medicinal plants of India. J Ethnopharmacol. 2007;114(2):103-113.

5. Enoch S, Leaper DJ. Basic science of wound healing. Surgery. 2005; 23(2):37-42.

6. Smith R. Recovery and tissue repair. Br Med Bull. 1985;41(3): 295-301.

7. Martin P, Hopkinson-Wooley J, McCluskey J. Growth factors and cutaneous wound repair. Prog Growth Factor Res. 1992;4(1): 25-44.

8. Martin P. Wound healing - aiming for perfect skin regeneration. Science. 1997;276(5309):75-81.

9. Gopinath D, Ahmed MR, Gomathi K, Chitra K, Sehgal PK, Jayakumar R. Dermal wound healing processes with curcumin incorporated collagen films. Biomaterials. 2004;25(10):1911-1917.

10. Souza SM, Aquino LC, Milach AC, Bandeira MA, Nobre ME, Viana GS. Antiinflammatory and antiulcer properties of tannins from Myracrodruon urundeuva Allemão (Anacardiaceae) in rodents. Phytother Res. 2007;21(3):220-225.

11. Majeed M, Bhat B, Jadhav AN, Srivastava JS, Nagabhushanam K. Ascorbic acid and tannins from Emblica officinalis Gaertn. Fruits - a revisit. J Agric Food Chem. 2009;57(1):220-225.

12. Tsala DE, Amadou D, Habtemariam S. Natural wound healing and bioactive natural products. Phytopharmacology. 2013;4(3):532-560.

13. Sharma RA, Gescher AJ, Steward WP. Curcumin: the story so far. Eur J Cancer. 2005;41(13):1955-1968.

14. Shen L, Ji HF. The pharmacology of curcumin: is it the degradation products? Trends Mol Med. 2012;18(3):138-144.

15. Sharma SP, Aithal KS, Srinivasan KK, et al. Antiinflammatory and wound healing activities of the crude alcoholic extract and flavonoids of Vitex leucoxylon. Fitoterapia. 1990;61(3):263-265.

16. Chithra P, Suguna L, Chandrakasan G. Influence of arginine in wound healing in rats. J Clin Biochem Nutr. 1995;18(2):111-117.

17. Sidhu GS, Singh AK, Thaloor D, et al. Enhancement of wound healing by curcumin in animals. Wound Repair Regen. 1998;6(2):167-177.

18. Panchatcharam M, Miriyala S, Gayathri VS, Suguna L. Curcumin improves wound healing by modulating collagen and decreasing reactive oxygen species. Mol Cell Biochem. 2006;290(1-2):87-96.

19. Basnet P, Skalko-Basnet N. Curcumin: an anti-inflammatory molecule from a curry spice on the path to cancer treatment. Molecules. 2011;16(6):4567-4598.

20. Mukherjee PK, Maity N, Nema NK, Sarkar BK. Bioactive compounds from natural resources against skin aging. Phytomedicine. 2011;19(1): 64-73.

21. Thangapazham RL, Sharad S, Maheshwari RK. Skin regenerative potentials of curcumin. Biofactors. 2013;39(1):141-149.

22. Nakamura Y, Ohto Y, Murakami A, Osawa T, Ohigashi H. Inhibitory effects of curcumin and tetrahydrocurcuminoids on the tumor promoterinduced reactive oxygen species generation in leukocytes in vitro and in vivo. Jpn J Cancer Res. 1998;89(4):361-370.

23. Pan MH, Huang TM, Lin JK. Biotransformation of curcumin through reduction and glucuronidation in mice. Drug Metab Dispos. 1999;27(4):486-494.

24. Karthikesan K, Pari L, Menon VP. Protective effect of tetrahydrocurcumin and chlorogenic acid against streptozotocin-nicotinamide generated oxidative stress induced diabetes. J Funct Foods. 2010;2(2): 134-142.

25. Wahlang B, Pawar YB, Bansal AK. Identification of permeabilityrelated hurdles in oral delivery of curcumin using the Caco-2 cell model. Eur J Pharm Biopharm. 2011;77(2):275-282.

26. Kharkwal H, Bala K, Joshi DD, Katare DP. Bioavailability enhancement of curcuminoids using natural polymer. Der Pharmacia Lettre. 2012;4(6):1698-1711. 
27. Manjunatha JR, Bettadaiah BK, Negi PS, Srinivas P. Synthesis of amino acid conjugates of tetrahydrocurcumin and evaluation of their antibacterial and anti-mutagenic properties. Food Chem. 2013; 139(1-4):332-338.

28. Prasad S, Tyagi AK, Aggarwal BB. Recent developments in delivery, bioavailability, absorption and metabolism of curcumin: the golden pigment from golden spice. Cancer Res Treat. 2014;46(1):2-18.

29. Yeh CC, Su YH, Lin YJ, et al. Evaluation of the protective effects of curcuminoid (curcumin and bisdemethoxycurcumin)-loaded liposomes against bone turnover in a cell-based model of osteoarthritis. Drug Des Devel Ther. 2015;9:2285-2300.

30. Anand P, Kunnumakkara AB, Newman RA, Aggarwal BB. Bioavailability of curcumin: problems and promises. Mol Pharm. 2007;4(6):807-818.

31. Shahani K, Panyam J. Highly loaded, sustained-release microparticles of curcumin for chemoprevention. J Pharm Sci. 2011;100(7): 2599-2609.

32. Bhaskar Rao A, Prasad E, Deepthi SS, Ansari IA. Synthesis and biological evaluation of glucosyl curcuminoids. Arch Pharm (Weinheim). 2014;347(11):834-839.

33. van de Sandt JJ, Meuling WJ, Elliott GR, Cnubben NH, Hakkert BC. Comparative in vitro-in vivo percutaneous absorption of the pesticide propoxur. Toxicol Sci. 2000;58(1):15-22.

34. Li J, Jiang Y, Wen J, Fan G, Wu Y, Zhang C. A rapid and simple HPLC method for the determination of curcumin in rat plasma: assay development, validation and application to a pharmacokinetic study of curcumin liposome. Biomed Chromatogr. 2009;23(11): 1201-1207.

35. Heath DD, Pruitt MA, Brenner DE, Rock CL. Curcumin in plasma and urine: quantitation by high-performance liquid chromatography. J Chromatogr B Analyt Technol Biomed Life Sci. 2003;783(1):287-295.

36. Mori R, Kondo T, Ohshima T, Ishida Y, Mukaida N. Accelerated wound healing in tumor necrosis factor receptor p55-deficient mice with reduced leukocyte infiltration. FASEB J. 2002;16(9):963-974.

37. Mustafa MR, Mahmood AA, Sidik K, Noor SM. Evaluation of wound healing potential of Ageratum conyzoides leaf extract in combination with honey in rats as animal model. Int J Mol Med Adv Sci. 2005; 1(4):406-410.
38. Hicks CN. Research Methods for Clinical Therapeutics. 3rd ed. Edinburgh: Churchill Livingstone; 1999.

39. Walker HL, Masson AD Jr. A standard animal burn. J Trauma. 1968; 8(6):1049-1051.

40. Lowry OH, Rosebrough NJ, Farr AL, Randall RJ. Protein measurement with the Folin phenol reagent. J Biol Chem. 1951;193(1):265-275.

41. Anderson SM, Elliott RJ. Evaluation of a new, rapid collagen assay. Biochem Soc Trans. 1991;19(4):389S.

42. McManus JFA, Mowry RW. Staining Methods, Histologic and Histochemical. New York, NY: Harper \& Row; 1965.

43. Singer AJ, Clark RAF. Cutaneous wound healing. N Engl J Med. 1999; 341(10):738-746.

44. Guo S, Dipietro LA. Factors affecting wound healing. J Dent Res. 2010; 89(3):219-229.

45. Kulac M, Aktas C, Tulubas F, et al. The effects of topical treatment with curcumin on burn wound healing in rats. J Mol Histol. 2013;44(1): 83-90.

46. Abramov Y, Golden B, Sullivan M, et al. Histological characterization of vaginal vs. abdominal surgical wound healing in a rabbit model. Wound Repair Regen. 2007;15(1):80-86.

47. Honardoust D, Tredget EE. Adult skin wounds can be induced to regenerate through modulation of cells and extracellular matrix molecules. Adv Wound Care. C. K. Sen, Ed., vol. 2, Mary Ann Liebert, 2011.

48. Hsu CH, Cheng AL. Clinical studies with curcumin. Adv Exp Med Biol. 2007;595:471-480.

49. Aggarwal BB, Kumar A, Bhharti AC, et al. Anticancer potential of curcumin: preclinical \& clinical studies. Anticancer Res. 2003; 23(1A):363-398.

50. Thangapazham RL, Sharma A, Maheshwari RK. Beneficial role of curcumin in skin diseases. Adv Exp Med Biol. 2007;595:343-357.

51. Majeed M, Badmaev V, inventors; Sabinsa Corporation, assignee. Cross-regulin composition of turmeric-derived tetrahydrocurcuminoids for skin lightening and protection against UVB rays. United States patent 6653327 B2. 2003 Nov 25. 


\section{Supplementary material}

Table SI Histopathological scoring

\begin{tabular}{|c|c|c|c|c|c|}
\hline \multirow[t]{2}{*}{ Group: test compound } & \multicolumn{5}{|c|}{ Histopathological scoring* } \\
\hline & Epithelialization & Angiogenesis & Fibroblasts & Collagen & $\begin{array}{l}\text { Macrophages/ } \\
\text { lymphocytes }\end{array}$ \\
\hline A: control & I & 3 & 3 & 1 & 3 \\
\hline B: curcumin $2 \%$ & I & 3 & 3 & 1 & 2 \\
\hline C: THC 2\% & 2 & 2 & 3 & 2 & 2 \\
\hline D: THC 3\% & 2 & 2 & 3 & 2 & 2 \\
\hline E: THC 5\% & 2 & 2 & 2 & 3 & 2 \\
\hline F: glucosyl-THC 2\% & 2 & 2 & 2 & 2 & 1 \\
\hline G: glucosyl-THC 3\% & 2 & I & 2 & 3 & 1 \\
\hline H: glucosyl-THC 5\% & 3 & 0 & 0 & 3 & 1 \\
\hline
\end{tabular}

Notes: *Abramov's histological scoring system was used for scoring epithelialization, fibrosis, angiogenesis, and collagen level.' Abramov's system assesses each parameter independently, giving each a score of $0-3$, as follows:

- Collagen: $0=$ none, $I=$ scant, $2=$ moderate, $3=$ abundant

- Epithelialization: $0=$ none, $I=$ partial, $2=$ complete but immature/thin, $3=$ complete and mature.

- Angiogenesis: $0=$ none, $I=\leq 5$ vessels per HPF, $2=6-10$ vessels per HPF, $3=>10$ vessels per HPF.

- Fibrosis: $0=$ none to minimal fibroblasts, $I=$ few fibroblasts, $2=$ more fibroblasts, $3=$ predominantly fibroblasts.

As well, the number of macrophages was scored, as: $1=0-25$ macrophages, $2=26-50$ macrophages, $3=>51$ macrophages.

Abbreviations: glucosyl-THC, glucosyl-conjugated tetrahydrocurcumin; HPF, high-power field; THC, tetrahydrocurcumin.

\section{Reference}

1. Abramov Y, Golden B, Sullivan M, et al. Histologic characterization of vaginal vs. abdominal surgical wound healing in a rabbit model. Wound Repair Regen. 2007;15(1):80-86.

\section{Publish your work in this journal}

Drug Design, Development and Therapy is an international, peerreviewed open-access journal that spans the spectrum of drug design and development through to clinical applications. Clinical outcomes, patient safety, and programs for the development and effective, safe, and sustained use of medicines are a feature of the journal, which has also been accepted for indexing on PubMed Central. The manuscript management system is completely online and includes a very quick and fair peer-review system, which is all easy to use. Visit http://www.dovepress.com/testimonials.php to read real quotes from published authors.

Submit your manuscript here: http://www.dovepress.com/drug-design-development-and-therapy-journal 